\title{
Percutaneous "edge-to-edge" leaflet repair in patients with secondary mitral valve regurgitation
}

\author{
Giulia Masiero, Giulio Rodinò, Giuseppe Tarantini \\ Department of Cardiac, Thoracic, Vascular Science and Public Health, University of Padova, Padova 35128, Italy.
}

Correspondence to: Dr. Giuseppe Tarantini, Department of Cardiac, Thoracic and Vascular Science, University of Padova, Via Giustiniani 2, Padova 35128, Italy. E-mail: giuseppe.tarantini.1@gmail.com

\begin{abstract}
How to cite this article: Masiero G, Rodinò G, Tarantini G. Percutaneous "edge-to-edge" leaflet repair in patients with secondary
\end{abstract} mitral valve regurgitation. Mini-invasive Surg 2020;4:71. http://dx.doi.org/10.20517/2574-1225.2020.56

Received: 8 Jun 2020 First Decision: 15 Jul 2020 Revised: 5 Aug 2020 Accepted: 18 Aug 2020 Published: 16 Oct 2020

Academic Editor: Azeem Latib Copy Editor: Cai-Hong Wang Production Editor: Jing Yu

\begin{abstract}
Functional or secondary mitral regurgitation (MR) is a heterogeneous entity afflicting patients with heart failure both with reduced or preserved left ventricular ejection fraction. It results from an imbalance between closing forces and tethering or pushing strengths acting on the valve in the absence of structural alterations of mitral valve (MV) apparatus. According to previous studies, more than $20 \%$ of patients with heart failure and reduced left ventricular ejection fraction have severe $M R$, even though the definition of the severity of the MV disease in this setting remains a debated issue due to the poor reproducibility of quantitative measurements and its dynamic nature, highly dependent on left ventricular loading conditions and performance in relation to optimization of medical treatment. Furthermore, it is still unclear whether MR is a direct contributor to a worse prognosis or merely a marker of severity of the disease affecting the left ventricle. Isolated MV surgery in these patients is burdened by significant operative mortality, high rates of recurrent MR and absence of proven survival benefit. In recent years, percutaneous treatment of functional MR arose as a viable and safe alternative to conventional surgery, proving capable of reducing symptoms and recurrent hospitalization rates for heart failure, and even improving prognosis in selected patients. In this review we will discuss the percutaneous treatment of functional MR through transcatheter "edge-to-edge" leaflet repair performed with the two systems currently available: the MitraClip System and the PASCAL Repair System, from available evidence to technical practice.
\end{abstract}

Keywords: Functional mitral valve disease, mitral regurgitation, mitral insufficiency, heart failure, MitraClip, PASCAL

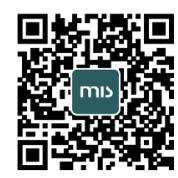




\section{INTRODUCTION}

Secondary mitral valve regurgitation (MR) is a heterogeneous entity afflicting patients with heart failure (HF) and is almost twice as common as the degenerative type. It occurs in up to $60 \%$ of patients after a myocardial infarction and is present in more than half of patients with dilated cardiomyopathy ${ }^{[1]}$. Despite its high prevalence, the optimal therapeutic apprsoach remains a matter of debate, with a disappointingly low level of evidence for guideline recommendations. The aim of this review is to portray the percutaneous treatment of functional MR through transcatheter "edge-to-edge" leaflet repair, from available evidence to technical practice.

\section{ETIOLOGY AND TREATMENT OF SECONDARY MR}

Functional MR results from an imbalance between closing forces and tethering or pushing strengths acting on the valve in the absence of structural alterations of mitral valve (MV) apparatus. It may occur in patients with heart failure both with reduced ejection fraction (HFrEF) or with preserved ejection fraction $(\mathrm{HFpEF})^{[2]}$. In the former setting, the underlying ischemic or non-ischemic triggers determine an eccentric left ventricle (LV) remodeling, either global or regional, with papillary muscle displacement resulting in enhanced tethering forces on the leaflets; on the other hand, ventricular dysfunction and annular dilatation determine a reduction in closing forces. The resulting secondary MR turns in chronic LV volume overload, thus inducing further remodeling and progression of the disease. In time, an LV diastolic dysfunction occurs, causing an increase in left atrial (LA) pressure and subsequent LA remodeling and enlargement with further annular dilatation, thus contributing to MV tenting ${ }^{[2,3]}$ [Figure 1]. More than $20 \%$ of patients with HFrEF have severe MR, with comparable rates between ischemic and non-ischemic etiologies ${ }^{[4]}$. Functional MR is also found in a significant proportion of patients with HFpEF but still little is known about its pathogenesis, clinical implications, and prognostic importance. Clinical conditions frequently associated in this setting include atrial fibrillation, severe aortic stenosis, diabetes mellitus and myocardial ischemic disease. Systolic MV tenting is supposedly due to LV diastolic disfunction causing increased LV and LA filling pressure, which in turn determines LA enlargement, annular dilatation, and leaflets malcoaptation ${ }^{[2,3]}$ [Figure 1]. Given the lack of data on prognosis, therapeutic options and management algorithms of MR in this clinical setting, this review will focus on the percutaneous treatment of MR in patients with HFrEF. In this setting, independent of the etiology of HFrEF, a higher degree of mitral regurgitation is strongly associated with poor clinical outcomes, even though it remains uncertain if it is a direct contributor to a worse prognosis or merely a marker of severity of the disease affecting the $\mathrm{LV}^{[5-7]}$. As a matter of fact, current European guidelines set lower thresholds for effective regurgitation orifice area (EROA) and regurgitant volume (RVol) to define severe secondary $\mathrm{MR}\left(\mathrm{EROA} \geq 20 \mathrm{~mm}^{2}\right.$; RVol $\geq 30 \mathrm{~mL}$ ) compared to that used for primary MR (EROA $\geq 40 \mathrm{~mm}^{2}$; RVol $\left.\geq 60 \mathrm{~mL}\right)^{[1,8,9]}$. However, the definition of the severity of $\mathrm{MV}$ disease remains a debated issue due to the poor reproducibility of EROA measurements, the close dependence of the EROA and RVol values on LV volume, and lastly, the dynamic change of LV loading conditions and performance after optimization of medical treatment ${ }^{[1,8,10-12]}$ [Table 1]. Therefore, a careful integration of quantitative, semiquantitative and qualitative echocardiographic parameters and clinical data, is necessary for deciding appropriate treatment. Different from primary MR, there is no evidence that surgical correction of valvular disease improves survival. Therefore, surgery is recommended only for patients with symptomatic severe MR and a left ventricular ejection fraction (LVEF) $>30 \%$ undergoing coronary artery by-bass graft, or in patients with lower EF but with evidence of myocardial viability and an option for revascularization. Isolated MV surgery is burdened by significant operative mortality, high rates of recurrent MR, absence of proven survival benefit and it may be considered in low surgical risk patients remaining symptomatic despite optimal medical management, including cardiac resynchronization therapy (CRT) with no markedly reduced LV function ${ }^{[1]}$. In cases of suitable valve morphology, transcatheter treatment remains an opportunity for higher surgical risk patients or for those without a revascularization option, after careful Heart-Team evaluation. Based on current clinical guidelines, transcatheter repair of 
A

Local LV remodellin Due to infero-posterior scar $\pm$

Papillary muscle dysfunction
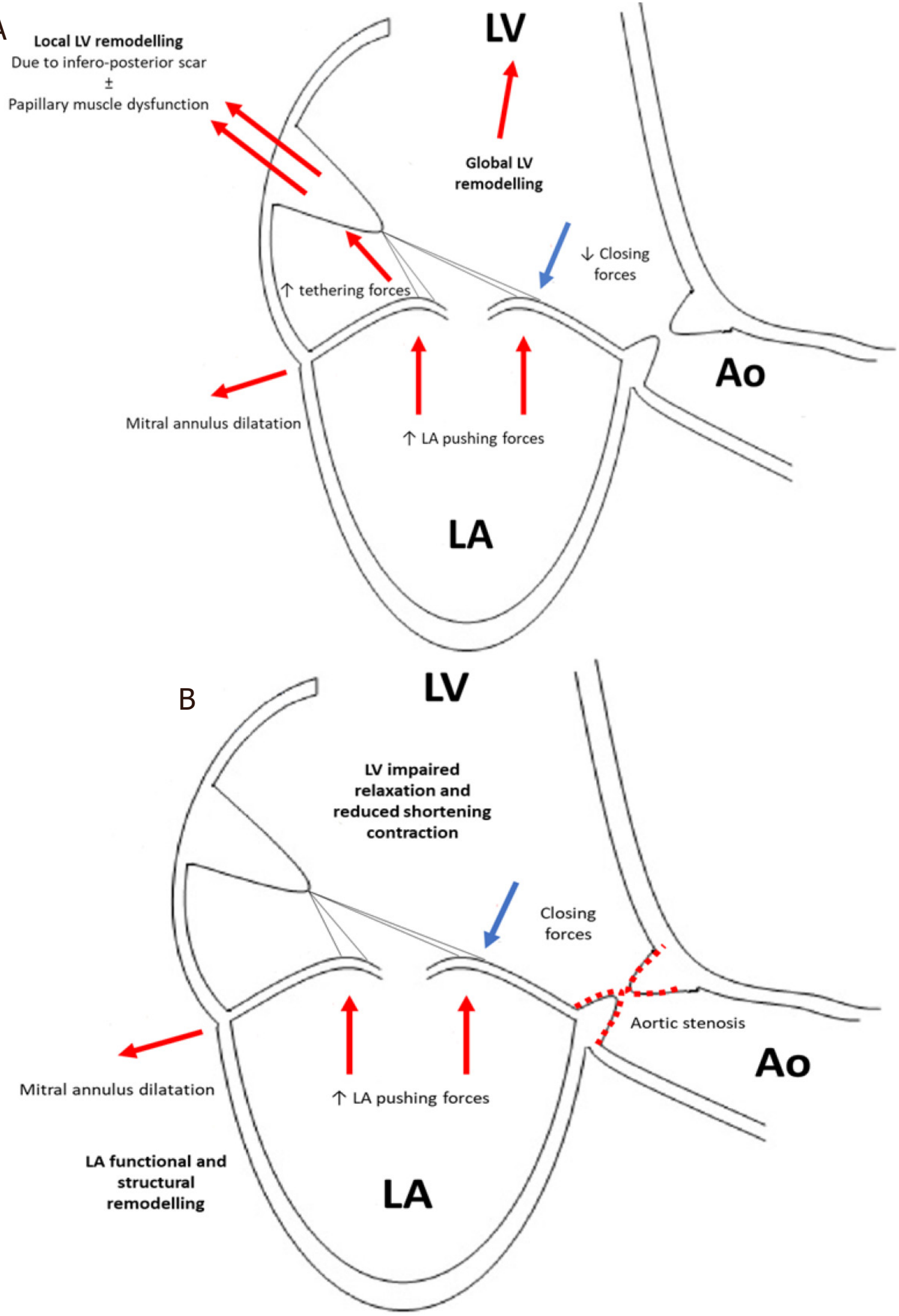

Figure 1. Underlying mechanisms of functional MR in HFrEF (A) and HFpEF (B). In HFpEF aortic stenosis may be one of the underlying conditions causing LV impaired relaxation and reduced shortening contraction. Other frequent conditions are diabetes mellitus and myocardial ischemic disease. Another possible condition is atrial fibrillation which, instead, directly causes LA functional and structural remodelling. MR: mitral regurgitation; LA: left atrium; LV: left ventricle; Ao: aorta 
Table 1. Association between MR severity and prognosis in real-world registries

\begin{tabular}{|c|c|c|c|c|c|c|}
\hline Author & No. of pts. & Type of study & LVEF cut-off & Etiology of MR & $\begin{array}{l}\text { Method of } \\
\text { grading MR }\end{array}$ & $\begin{array}{c}\text { MR as independent predictor } \\
\text { of mortality }\end{array}$ \\
\hline Grigioni et al. ${ }^{[6]}$ & 303 & $\begin{array}{l}\text { Single center, } \\
\text { Observational }\end{array}$ & N/A & $\begin{array}{l}\text { Ischemic (post- } \\
\mathrm{MI} \text { ) }\end{array}$ & QD, PISA & $E R O A \geq 20 \mathrm{~mm}^{2}$ \\
\hline Lancellotti et al. ${ }^{[7]}$ & 98 & $\begin{array}{l}\text { Single center, } \\
\text { Observational }\end{array}$ & $<45 \%$ & Ischemic & PISA & $E R O A \geq 20 \mathrm{~mm}^{2}$ \\
\hline Rossi et al. ${ }^{[5]}$ & 1,256 & $\begin{array}{l}\text { Multicenter, } \\
\text { Observational }\end{array}$ & N/A & $\begin{array}{l}\text { FMR } \\
62 \% \text { ischemic }\end{array}$ & VCW, PISA & $\begin{array}{l}E R O A \geq 20 \mathrm{~mm}^{2} \\
V C W>0.4 \mathrm{~cm}\end{array}$ \\
\hline Patel et al. ${ }^{[10]}$ & 558 & $\begin{array}{l}\text { Single center, } \\
\text { Observational }\end{array}$ & $<35 \%$ & $\begin{array}{l}\text { FMR } \\
54 \% \text { ischemic }\end{array}$ & PISA & $\begin{array}{l}\text { No difference for } E R O A \geq \text { or }< \\
20 \mathrm{~mm}^{2}\end{array}$ \\
\hline Grayburn et al. ${ }^{[11]}$ & 336 & $\begin{array}{l}\text { Substudy of } \\
\text { multicenter RCT }\end{array}$ & $<35 \%$ & $\begin{array}{l}\text { FMR } \\
57 \% \text { ischemic }\end{array}$ & VCW, QD, PISA & $\begin{array}{l}\text { MR not a predictor; } V C W \geq \\
0.4 \mathrm{~cm} \text { only for a composite EP }\end{array}$ \\
\hline
\end{tabular}

Pts.: patients; LVEF: left ventricular ejection fraction; N/A: not available; QD: quantitative Doppler; PISA: proximal isovelocity surface area; VCW: vena contracta width; EROA: effective regurgitant orifice area; RCT: randomized clinical trial; EP: endpoint; MR: mitral regurgitation

the MV may be considered as a second or third-line therapy in symptomatic (New-York Heart Association functional class $\geq 2$ ) patients with HFrEF and one of the following ${ }^{[1,9]}$ :

(1) Severe functional MR despite maximum tolerated medical therapy, with no indication to CRT or heart transplant/left ventricular assist device (HT/LVAD) and high surgical risk due to old age, frailty, or severe comorbidity.

(2) Severe MV disease despite optimal medical therapy and non-responder to CRT, with high surgical risk and no indication to HT/LVAD.

(3) Severe secondary MR despite maximum tolerated medical therapy, non-responder to CRT and with an indication to HT, as a "bridge therapy".

The MitraClip System (Abbott Vascular, Santa Clara, CA, USA) is the most investigated and adopted device for percutaneous "edge-to-edge" valve repair in functional MV disease. Despite being less effective than conventional surgery in reducing MR, it has achieved higher safety, similar improvements in clinical outcomes and a survival benefit compared to medical therapy, as recent studies have shown ${ }^{[13-15]}$. However, the conflicting results of the latest two major trials investigating the use of MitraClip in secondary MR highlighted a great debate on optimal patient selection criteria for this procedure ${ }^{[14,15]}$. Adjunctively, the new PASCAL repair system (Edwards Lifesciences, Irvine, CA, USA) recently received CE-mark for the treatment of functional and degenerative MR [Figure 2].

\section{TECHNICAL ASPECTS OF THE TRANSCATHETER MV “EDGE-TO-EDGE” LEAFLET REPAIR FOR THE TREATMENT OF FUNCTIONAL MR}

Patient selection for the percutaneous "edge-to-edge" procedure is currently performed by pre-operative multi-modality imaging assessment, using both $2 \mathrm{D}$ and $3 \mathrm{D}$ transthoracic echocardiography (TTE) and transesophageal echocardiography (TEE). TEE allows for confirmation and severity assessment of secondary MR, as well as evaluation of the anatomic suitability for a MitraClip implantation. Currently, the main exclusion criteria include a very short posterior leaflet $(<7 \mathrm{~mm})$, a mitral valve area $<3 \mathrm{~cm}^{2}$, the presence of severe calcification of the leaflets in the grasping area and a combined MV disease on rheumatic basis. As stated before, since MR is a dynamic condition, it is necessary to perform the preoperative evaluation in the best hemodynamic conditions possible, with normal blood pressure and heart rate following optimization of medical therapy.

In the last decade, thousands of patients treated with the MitraClip System achieved significant improvements in symptoms, functional status and quality of life, favorable LV remodeling and a reduction of HF hospitalizations ${ }^{[13,16]}$. This device reproduced the surgical "edge-to-edge" repair of the MV through a 

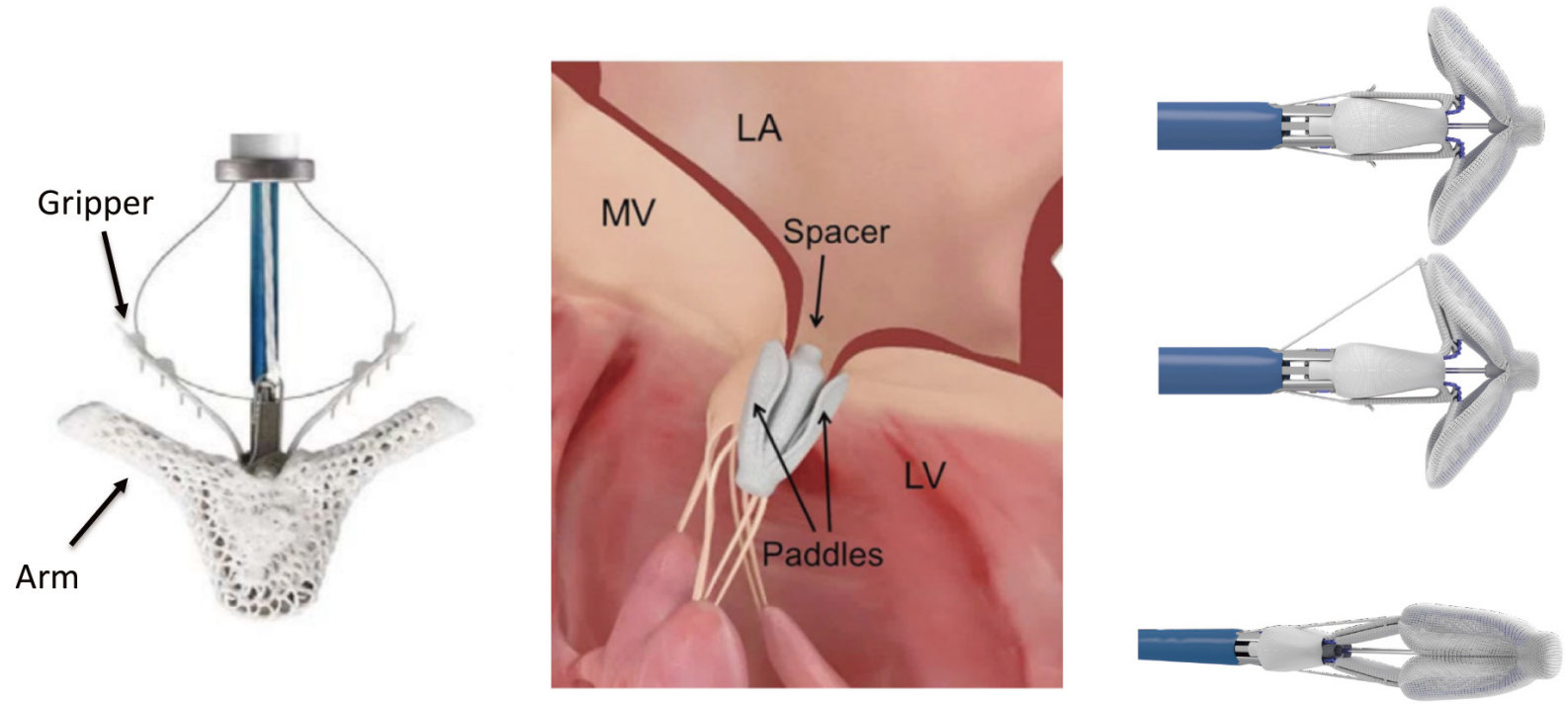

Figure 2. The PASCAL implant consists of a $10 \mathrm{~mm}$ central nitinol woven spacer that acts as a filler in the regurgitant orifice of the $\mathrm{MV}$, and in its closed conformation is attached to the valve leaflets by two paddles and clasps. Differently from the MitraClip system, each of the 2 clasps can be activated independently and the device can take an elongated form. LA: left atrium; LV: left ventricle; MV: mitral valve

percutaneous approach, producing a "double-orifice" valve and reducing the severity of the regurgitation. The steerable 24 Fr catheter with a clip delivery system on its proximal end clip is advanced in the LA through a transseptal approach using a venous femoral access, and then further advanced in the LV to effectively grasp together the MV leaflets. The whole procedure is carried out under general anesthesia and with fluoroscopic and TEE imaging guidance. As opposed to the MitraClip system, the PASCAL implant consists of a $10 \mathrm{~mm}$ central nitinol woven spacer that acts as a filler in the regurgitant orifice of the MV, and in its closed conformation is attached to the valve leaflets by two paddles and clasps [Figure 2]. The paddles, which rest on the ventricular side of the valve leaflets, secure the leaflets against the nitinol spacer and ensure a low and homogeneous pressure distribution on the valve tissue. Furthermore, the paddles flex on every heart beat so that the system dynamically flexes as the valve opens and closes, preserving the native anatomical geometry. Using the PASCAL system, each of the 2 clasps can be activated independently, so that tissue insertion between the paddles and the spacer can be optimized, improving results, allowing distribution of the traction on valve leaflets while maintaining a larger mitral orifice. Furthermore, low mitral gradients are ensured even in the case that two devices are needed. The PASCAL system consists of a steerable guide sheath intended to provide height on the mitral annulus plane, a steerable catheter allowing access to different locations across the coaptation line and an implant catheter that extends from the left atrium into the LV, used to deliver the implant. The three independent catheter movements in all planes allow for an easy height compensation in cases of sub-optimal trans-septal crossing, an intuitive control of the delivery system and a very simple positioning and orientation of the implant. Therefore, it is intended to assist the operator in the treatment of challenging anatomies, such as short posterior leaflets, large flail gaps, severe tethering, and severe annular dilatation. However, further studies are needed to assess the safety and effectiveness of this novel device in this complex anatomical setting.

Standard medical treatment to prevent clinically relevant stroke after percutaneous edge-to-edge MV repair is still a debated issue, due to the lack of dedicated randomized clinical trials aimed at comparing different treatment strategies. In the EVEREST II trial, patients were treated with heparin during the procedure, and a combination of aspirin (at a dose of $325 \mathrm{mg}$ daily) for 6 months and clopidogrel (at a dose of $75 \mathrm{mg}$ daily) for 30 days after the procedure ${ }^{[16]}$. Therefore, in the absence of risk factors requiring antithrombotic therapy such as atrial fibrillation, dual antiplatelet therapy using aspirin and clopidogrel for up to 6 months 
is currently the preferred therapeutic strategy ${ }^{[17]}$. However, recent observational studies suggest that temporary oral anticoagulation, with coumadin or apixaban, might be an effective strategy to reduce the incidence of stroke within the first 30 days after the MitraClip procedure in patients with maintained sinus rhythm, without an increase in minor and major bleeding events ${ }^{[18,19]}$. Adjunctive data relevant to medical treatment after a PASCAL implantation procedure are inadequate. Further studies should be conducted to address this important issue.

\section{EVIDENCE ON PERCUTANEOUS “EDGE-TO-EDGE” LEAFLET TREATMENT IN SECONDARY}

\section{MR}

Data on safety and effectiveness of the MitraClip device for the treatment of functional MR mainly result from two real-word prospective European multicenter registries and the two latest major trials ${ }^{[14,15,20,21]}$.

The ACCESS-EU study enrolled a total of 567 elderly patients with significant MR between 2009 and 2011, of which $79 \%$ presented with secondary MR, 85\% with NYHA class III or IV, and 53\% with LVEF $\leq 40 \%$. Acute procedural success occurred in $99.6 \%$ of patients. The survival rate at 1 -year follow-up was $82 \%$ with low rate of subjects (6\%) requiring MV surgery. Moreover, there was significant clinical improvement, with durable residual $\mathrm{MR}<2+$ and NYHA class I/II in the majority of patients (respectively $79 \%$ and $71 \%$ ) with a higher six-minute-walking-test and Minnesota-living-with-heart-failure score performance ${ }^{[20]}$.

In the following two years, the SENTINEL registry enrolled 628 patients with a mean age of $74 \pm 10$ years, lower than that of the ACCESS-EU study. Once again, functional MR was the prevalent pathogenesis (72\%). Acute procedural success was high (95\%) with only one clip implanted in two thirds of the population. The 1 -year mortality was comparable to previous studies (15\%), with a significantly higher rate of rehospitalization compared to the degenerative group $(26 \% v s .12 \%, P=0.009)$. Echocardiographic followup data showed a persistent reduction in the degree of mitral regurgitation at 1 year, with $6.0 \%$ of patients with residual severe $\mathrm{MR}^{[21]}$.

More recently, two large randomized clinical trials compared the MitraClip procedure to conservative treatment ${ }^{[14,15]}$. Despite their similarities, the studies showed conflicting results and conclusions, fostering the debate about the potential association between this transcatheter mitral repair and a significant survival benefit. Both MITRA-FR and COAPT were multicenter, randomized, open-label trials that enrolled 304 and 614 high surgical risk patients with HFrEF and symptomatic moderate-to-severe or severe functional MR respectively, with comparable rates of ischemic and non-ischemic etiologies. However, different from the first study, the latter involved higher volume centers and greater performance, performed a more rigorous clinical and instrumental patient selection, and proved a more careful medical therapy uptitration. At 12 months follow-up, the rate of the primary outcome (death from any cause or unplanned hospitalization for HF) was comparable between the two treatment groups of the MITRA-FR (55\% in the interventional group vs. $51 \%$ in the control group, $P=0.53$ ) with no significative difference among the individual components of the composite endpoint ${ }^{[14]}$. Conversely, the primary outcome in the COAPT trial consisted of all hospitalizations for HF within 24 months of follow-up, showing a significantly lower annualized rate in the device group as compared with the control group (respectively, 36\% per patient-year vs. $68 \%$ per patient-year, $P<0.001$ ). Moreover, the secondary endpoint of death from any cause within 24 months occurred in $29 \%$ of the patients in the MitraClip group as compared with $46 \%$ in the conservative group $(P<0.001)$. These clinical improvements and benefits were consistent across numerous subgroups, including patients who had ischemic MR etiology and non-ischemic cardiomyopathy, and were independent of the MR grade and LV volume and function at baseline ${ }^{[15]}$. Therefore, several authors highlighted that the studies indeed enrolled 2 distinctly different groups of patients; as a matter of fact, the patients enrolled in the COAPT trial had an almost 30\% higher mean EROA with an approximately $30 \%$ 

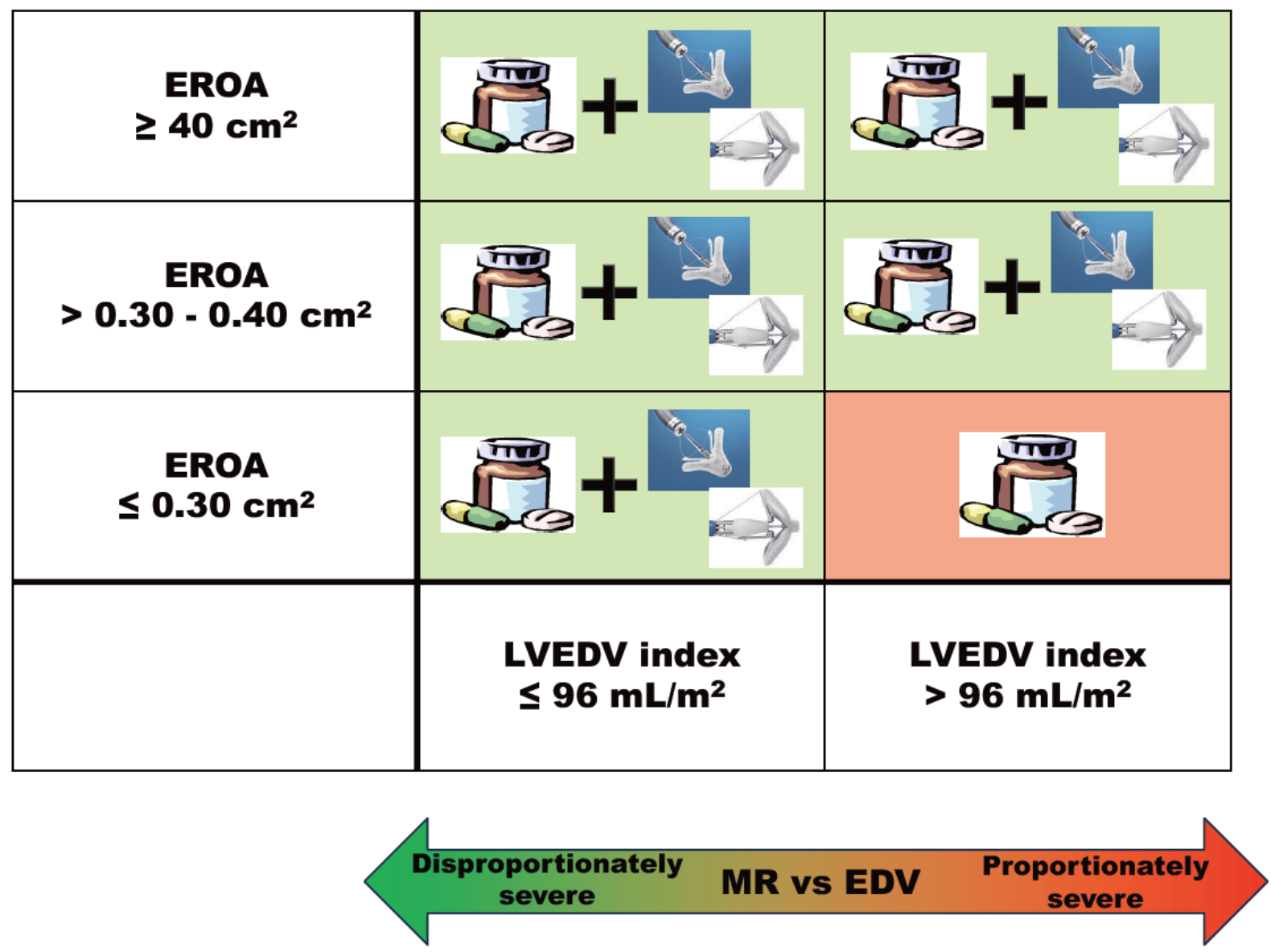

Figure 3. Treatment option (OMT, TMVR) of functional MR in patients with disproportionate or proportionate severe MR according to EROA and LVEDV. Based on an analysis of subgroups of patients enrolled in the COAPT trial and the MITRA-FR trial by M. Packer and P.A. Grayburn ${ }^{[26]}$. EROA: effective regurgitant orifice area; LVEDV: left ventricular end-diastolic volume; MR: mitral regurgitation; TMVR: transcatheter mitral valve repair; OMT: optical medical therapy. Copyright of the figure belongs to Prof. Tarantini

smaller average LVEDV as compared with those in the MITRA-FR trial. Furthermore, by stratifying the COAPT study population according to the level of EROA, only those patients with an EROA $<30 \mathrm{~mm}^{2}$ in the setting of a dilated LV $\left(>96 \mathrm{~mL} / \mathrm{m}^{2}\right)$ did not benefit from the MitraClip procedure. It has been speculated that the survival benefit shown in the COAPT study was strictly dependent on the selection of patients with a greater degree of MR, disproportionately higher than the amount of LV enlargement, and the exclusion of those with a more advanced stage of LV disease [Figure 3] ${ }^{[22]}$. Further data on the treatment of functional MR in HFrEF patients with the MitraClip System will be provided by two ongoing RCTs: the Reshape-HF2 trial (NCT02444338) and the MATTERHORN trial (NCT02371512).

Current evidence about the PASCAL device is limited to the multicenter CLASP CE-Mark study, a prospective, single-arm study involving 62 patients with moderate-to-severe or severe MR and up to $52 \%$ with functional etiology ${ }^{[23-25]}$. Preliminary data showed encouraging results with a $95 \%$ successful implantation rate, low major adverse event rate at 30 days, and sustained clinical improvement at 6-month and 1-year follow-ups in terms of MR grade reduction, NYHA functional class, 6-minute walk distance and Kansas City Cardiomyopathy Questionnaire and EQ-5D scores gain. Further data will be provided by the Edwards PASCAL CLASP IID/IIF Pivotal Clinical Trial (CLASP IID/IIF; NCT03706833), a prospective, multicenter, randomized, controlled trial comparing this novel device to the MitraClip. 

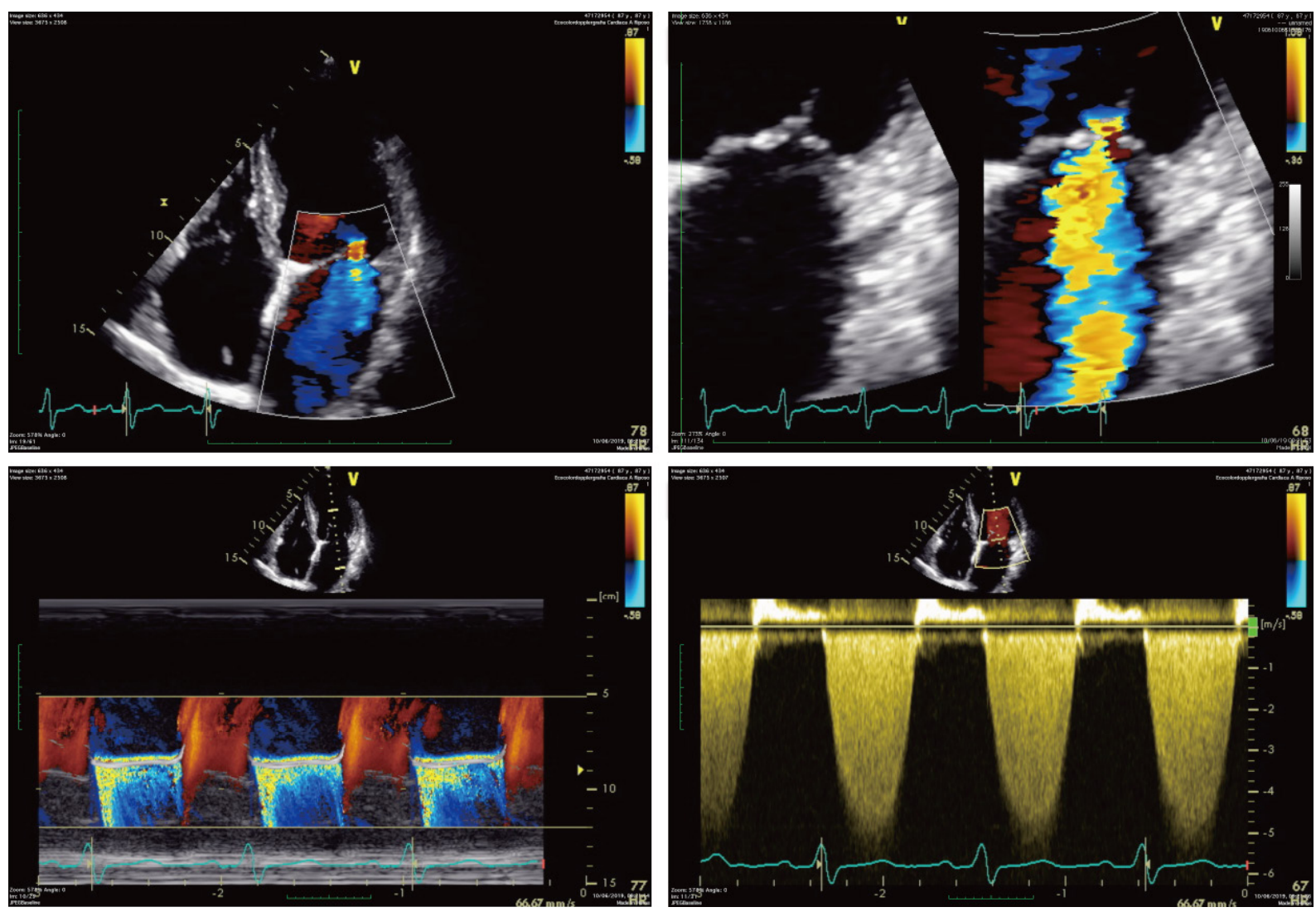

Figure 4. Pre-procedural transthoracic Doppler echocardiography evaluation of the mitral valve showing severe functional mitral regurgitation with a holosystolic central jet in a dilated cardiomyopathy (in particular: posterior leaflet of $8 \mathrm{~mm}$, an effective orifice area of $30 \mathrm{~mm}^{2}$ and a mitral valve area of $5 \mathrm{~cm}^{2}$ )

\section{CASE EXAMPLE: SECONDARY MR TREATED WITH THE PASCAL SYSTEM}

We report a case of an 88-year-old male with a history of ischemic cardiopathy already treated with percutaneous coronary intervention on left anterior descending coronary artery and right coronary artery, previous pace-maker implantation, severe chronic nephropathy and obstructive pulmonary disease. He progressively developed a severe functional MR with NYHA functional class II-III despite optimal medical therapy; a CRT therapy was not indicated and the previous stents on LAD and RCA were well working at the follow-up coronary angiography. The MV disease severity was evaluated on the basis of and confirmed by TEE. The $2 \mathrm{D}$ and $3 \mathrm{D}$ TTE showed a moderate reduction of the LVEF, regional wall motion abnormalities and preserved ventricular dimension, a normal right ventricular systolic function with mild pulmonary hypertension, a severe LA dilatation and a severe functional MR with a holosystolic central jet due to the tethering of a short posterior leaflet $(8 \mathrm{~mm})$, an effective orifice area of $30 \mathrm{~mm}^{2}$ and a MV area of $5 \mathrm{~cm}^{2}$ [Figure 4]. Given the advanced age and frailty, after the TEE assessment, a multidisciplinary HeartTeam defined the patient a suitable candidate for percutaneous "edge-to-edge" repair with the PASCAL repair system. The major MR jet was across A2-P2 segment so the intended implantation strategy was one central PASCAL device. A trans-septal puncture was done, aiming for a posterosuperior position in the fossa ovalis and with a measured height of 4.0-4.5 cm over the atrio-ventricular plan. Under TEE and fluoroscopic guidance, the steerable guide sheath and the steerable catheter were introduced into the LA while simultaneously flexing the implant catheter towards the MV. The valve was crossed with the opened paddles, achieving a straightforward position below the leaflets. After the 3D-TEE orthogonal alignment to the coaptation mitral line [Video 1], the implant was retracted until the leaflets were grasped simultaneously and thereafter optimized independently to place the leaflet deeper and its tip closer to the spacer 

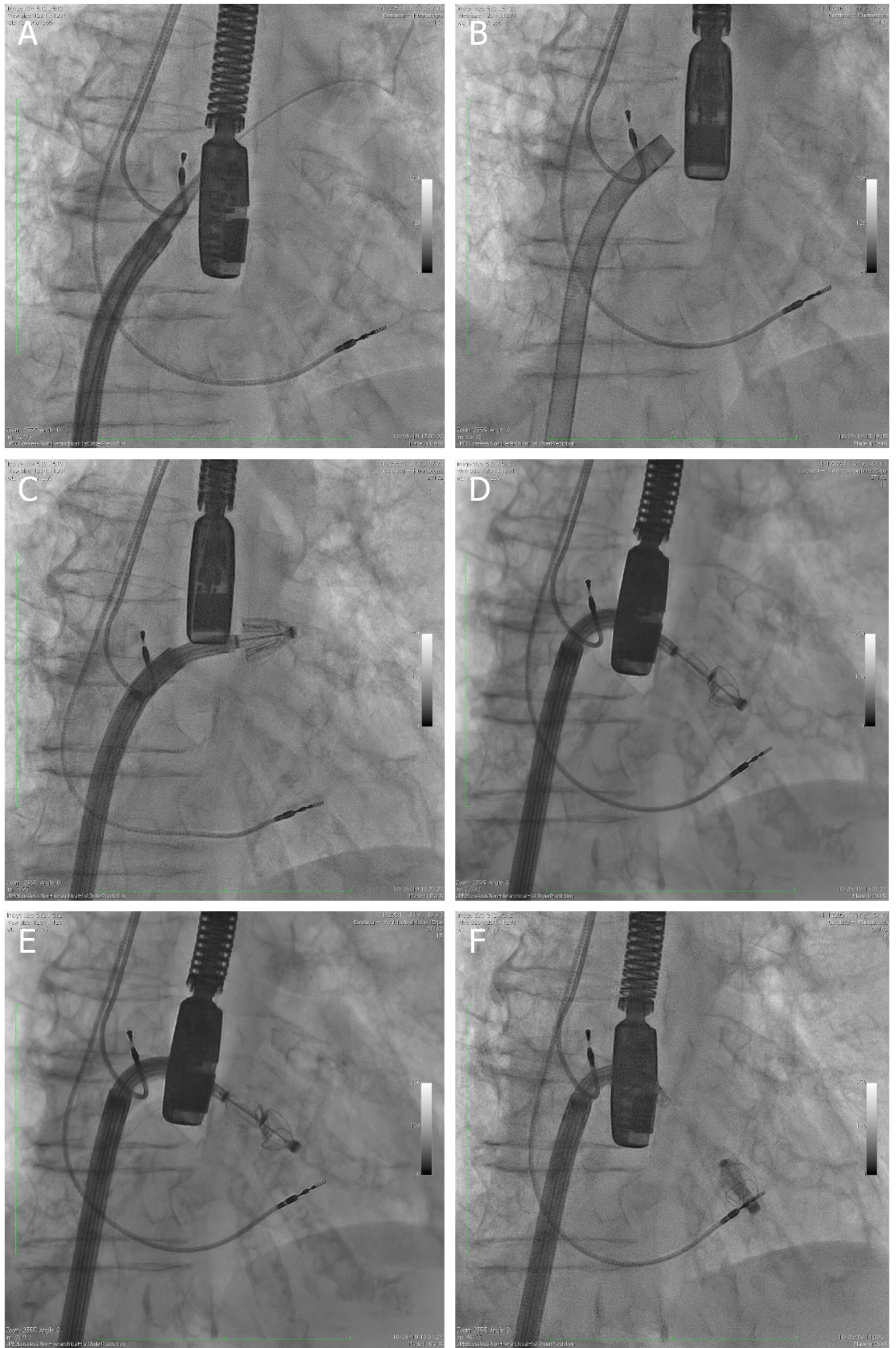

Figure 5. Procedural fluoroscopic steps for the implantation of the Edwards PASCAL transcatheter mitral valve repair system. With a transeptal approach, the steerable guide sheath and the steerable catheter were introduced into the left atrium while simultaneously flexing the implant catheter toward the mitral valve (A-C); the valve was crossed with the opened paddles achieving a straightforward positioning below the leaflets. After the three-dimensional transesophageal echocardiography guided orthogonal alignment to the coaptation mitral line, the implant was retracted until leaflets were grasped simultaneously (D, E); when sufficient and straight leaflet insertion was confirmed, the clasps were dropped, and device was closed. Immediately after, the residual mitral regurgitation and transvalvular gradient were systematically assessed to confirm optimal mitral regurgitation reduction before final deployment (F) 

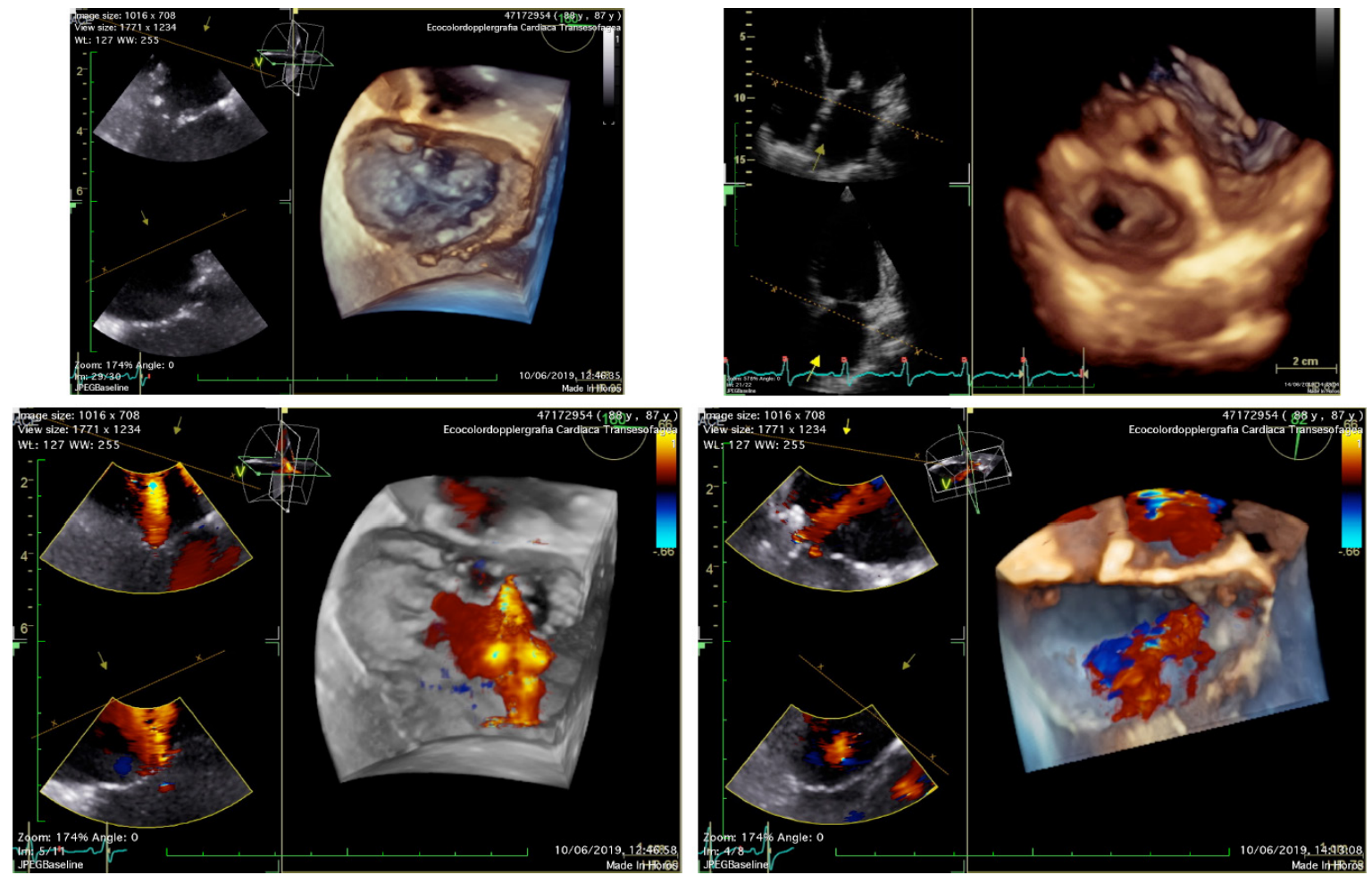

Figure 6. Periprocedural three-dimensional transesophageal echocardiography evaluation of mitral valve showing a pre-procedural severe functional mitral regurgitation with a holosystolic central jet and a consistent post-procedural reduction of the mitral regurgitation grade with the typical "double-orifice" valve
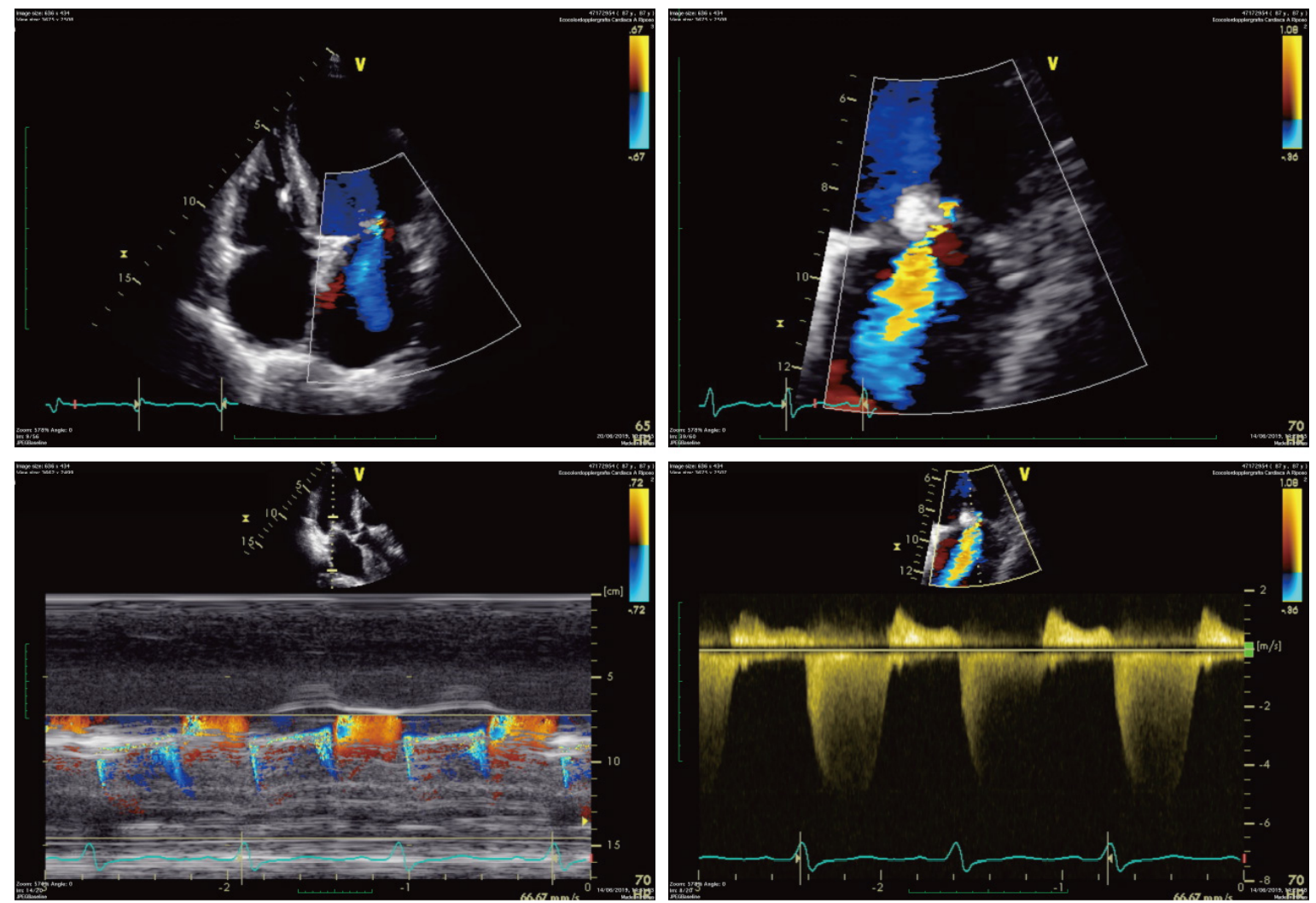

Figure 7. Post-procedural transthoracic Doppler echocardiography mitral valve evaluation showing mild regurgitation with acceptable mitral gradient (in particular: mean MV gradient of $3 \mathrm{mmHg}$, an effective orifice area of $14 \mathrm{~mm}^{2}$ and a MV area of $3 \mathrm{~cm}^{2}$ ) 
[Videos 2 and 3]. When sufficient and straight leaflet insertion was confirmed, the clasps were dropped, and device was closed. Immediately after, the residual MR and transvalvular gradient were systematically assessed to confirm optimal MR reduction before final deployment [Figures 5 and 6] [Video 4]. Remarkably, a drop in the mean left-atrial pressure from 16 to $8 \mathrm{mmHg}$ was observed. Mild regurgitation was confirmed at TTE before discharge (in particular: residual mean MV gradient of $3 \mathrm{mmHg}$, effective orifice area of $14 \mathrm{~mm}^{2}$ and MV area of $3 \mathrm{~cm}^{2}$ ) [Figure 7] and at 30-days follow-up with acceptable mitral gradient and clinical improvement (NYHA class I-II).

\section{CONCLUSION}

The percutaneous treatment of functional MR through transcatheter "edge-to-edge" leaflet repair has recently risen as a viable and safe alternative to conventional surgery in selected patients with severe disease who remain symptomatic despite maximally tolerated guideline-directed medical therapy and judged at high surgical risk by a multidisciplinary and experienced heart team. Two devices are currently available, the MitraClip and the PASCAL Repair Systems, with peculiar technical aspects and evidence.

\section{DECLARATIONS}

\section{Authors' contributions}

Participated to the conception and the drafting of the manuscript, its critical revision for important intellectual content and the final approval of the submitted text; agreement for all aspects of the work ensuring that questions related to the accuracy or integrity of any part of the work are appropriately investigated and resolved: Masiero G, Rodinò G, Tarantini G

\section{Availability of data and materials}

Not applicable.

\section{Financial support and sponsorship}

None.

\section{Conflicts of interest}

Tarantini G reports honoraria for lectures from Abbott Vascular and Edwards Lifesciences; Masiero G and Rodinò $\mathrm{G}$ declared that there are no conflicts of interest.

\section{Ethical approval and consent to participate}

Not applicable.

\section{Consent for publication}

Not applicable.

\section{Copyright}

(c) The Author(s) 2020.

\section{REFERENCES}

1. Baumgartner H, Falk V, Bax JJ, De Bonis M, Hamm C, et al; ESC Scientific Document Group. 2017 ESC/EACTS Guidelines for the management of valvular heart disease. Eur Heart J 2017;38:2739-91.

2. Ennezat PV, Maréchaux S, Pibarot P, Le Jemtel TH. Secondary mitral regurgitation in heart failure with reduced or preserved left ventricular ejection fraction. Cardiology 2013;125:110-7.

3. Inciardi RM, Rossi A, Benfari G, Cicoira M. Fill in the gaps of secondary mitral regurgitation: a continuum challenge from pathophysiology to prognosis. Curr Heart Fail Rep 2018;15:106-15.

4. Iung B, Vahanian A. Prevalence and definition of secondary mitral regurgitation. In: Fattouch K, Lancellotti P, Angelini GD, editors. Secondary Mitral Valve Regurgitation. London: Springer; 2015. pp. 1-6. 
5. Rossi A, Dini FL, Faggiano P, Agricola E, Cicoira M, et al. Independent prognostic value of functional mitral regurgitation in patients with heart failure. A quantitative analysis of 1256 patients with ischaemic and non-ischaemic dilated cardiomyopathy. Heart 2011;97:1675-80.

6. Grigioni F, Enriquez-Sarano M, Zehr KJ, Bailey KR, Tajik AJ. Ischemic mitral regurgitation: long-term outcome and prognostic implications with quantitative Doppler assessment. Circulation 2001;103:1759-64.

7. Lancellotti P, Troisfontaines P, Toussaint AC, Pierard LA. Prognostic importance of exercise-induced changes in mitral regurgitation in patients with chronic ischemic left ventricular dysfunction. Circulation 2003;108:1713-7.

8. Lancellotti P, Tribouilloy C, Hagendorff A, Popescu BA, Edvardsen T, et al; Scientific Document Committee of the European Association of Cardiovascular Imaging. Recommendations for the echocardiographic assessment of native valvular regurgitation: an executive summary from the European Association of Cardiovascular Imaging. Eur Heart J Cardiovasc Imaging 2013;14:611-44.

9. Nishimura RA, Otto CM, Bonow RO, Carabello BA, Erwin JP 3rd, et al. 2017 AHA/ACC Focused Update of the 2014 AHA/ACC Guideline for the Management of Patients With Valvular Heart Disease: A Report of the American College of Cardiology/American Heart Association Task Force on Clinical Practice Guidelines. J Am Coll Cardiol 2017;70:252-89.

10. Patel JB, Borgeson DD, Barnes ME, Rihal CS, Daly RC, et al. Mitral regurgitation in patients with advanced systolic heart failure. J Card Fail 2004;10:285-91.

11. Grayburn PA, Appleton CP, DeMaria AN, Greenberg B, Lowes B, et al; BEST Trial Echocardiographic Substudy Investigators. Echocardiographic predictors of morbidity and mortality in patients with advanced heart failure: the Beta-blocker Evaluation of Survival Trial (BEST). J Am Coll Cardiol 2005;45:1064-71.

12. Grayburn PA, Carabello B, Hung J, Gillam LD, Liang D, et al. Defining "severe" secondary mitral regurgitation: emphasizing an integrated approach. J Am Coll Cardiol 2014;64:2792-801.

13. Feldman T, Kar S, Elmariah S, Smart SC, Trento A, et al; EVEREST II Investigators. Randomized comparison of percutaneous repair and surgery for mitral regurgitation: 5-year results of EVEREST II. J Am Coll Cardiol 2015;66:2844-54.

14. Obadia JF, Messika-Zeitoun D, Leurent G, Iung B, Bonnet G, et al; MITRA-FR Investigators. Percutaneous repair or medical treatment for secondary mitral regurgitation. N Engl J Med 2018;379:2297-306.

15. Stone GW, Lindenfeld J, Abraham WT, Kar S, Lim DS, et al; COAPT Investigators. Transcatheter mitral-valve repair in patients with heart failure. N Engl J Med 2018;379:2307-18.

16. Feldman T, Foster E, Glower DD, Kar S, Rinaldi MJ, et al; EVEREST II Investigators. Percutaneous repair or surgery for mitral regurgitation. N Engl J Med 2011;364:1395-406.

17. Capodanno D, Tamburino C. Antithrombotic strategies in valvular and structural heart disease interventions: current status and future directions. Interv Cardiol Clin 2013;2:635-42.

18. Geis N, Raake P, Kiriakou C, Mereles D, Frankenstein L, et al. Temporary oral anticoagulation after MitraClip - a strategy to lower the incidence of post-procedural stroke? Acta Cardiol 2020;75:61-7.

19. Seeger J, Markovic S, Kessler M, Rottbauer W, Wöhrle J. Apixaban after percutaneous Edge-to-Edge mitral valve repair in patients with maintained sinus rhythm. JACC Cardiovasc Interv 2019;12:214-6.

20. Maisano F, Franzen O, Baldus S, Schäfer U, Hausleiter J, et al. Percutaneous mitral valve interventions in the real world: early and 1-year results from the ACCESS-EU, a prospective, multicenter, nonrandomized post-approval study of the MitraClip therapy in Europe. J Am Coll Cardiol 2013;62:1052-61.

21. Nickenig G, Estevez-Loureiro R, Franzen O, Tamburino C, Vanderheyden M, et al; Transcatheter valve treatment sentinel registry investigators of the EURObservational research programme of the european society of cardiology. Percutaneous mitral valve edge-toedge repair: in-hospital results and 1-year follow-up of 628 patients of the 2011-2012 Pilot European Sentinel Registry. J Am Coll Cardiol 2014;64:875-84.

22. Grayburn PA, Sannino A, Packer M. Proportionate and disproportionate functional mitral regurgitation: a new conceptual framework that reconciles the results of the MITRA-FR and COAPT trials. JACC Cardiovasc Imaging 2019;12:353-62.

23. Lim DS, Kar S, Spargias K, Kipperman RM, O’Neill WW, et al. Transcatheter valve repair for patients with mitral regurgitation: 30-day results of the CLASP study. JACC Cardiovasc Interv 2019;12:1369-78.

24. Mazimba S, Lim SD, Kipperman R, Spargias K, Kar S, et al. Six-month outcomes from the multicenter, prospective study with the novel PASCAL Transcatheter Valve Repair System for Patients with Mitral Regurgitation in the CLASP Study. J Card Fail 2019;25:S6.

25. Kar S, Ng M, Lim S, Walters D, Webb J, et al. TCT-91 1-year outcomes from the multicenter, prospective study with the novel PASCAL transcatheter valve repair system for patients with mitral regurgitation in the CLASP study. J Am Coll Cardiol 2019;74:B91.

26. Packer M, Grayburn PA. New evidence supporting a novel conceptual framework for distinguishing proportionate and disproportionate functional mitral regurgitation. JAMA Cardiol 2020:469. 